\title{
CONTRIBUCIÓN AL CONOCIMIENTO DE ENEMIGOS NATURALES DEL CHAPULÍN (ORTHOPTERA: ACRIDOIDEA) EN EL ESTADO DE QUERÉTARO, MÉXICO
}

\author{
Eliseo URIBE-GONZÁLEZ \& Miguel Ángel SANTIAGO-BASILIO \\ Comité Estatal de Sanidad Vegetal de Querétaro, A. C. Calamanda de Juárez, El Marques, Querétaro, \\ México. km.186.8 Autopista México-Querétaro. C.P.76247.<euribe@cesaveq.org.mx>
}

Uribe-González, E. \& M. A. Santiago-Basilio. 2012. Contribución al conocimiento de enemigos naturales del chapulín (Orthoptera: Acridoidea) en el estado de Querétaro, México. Acta Zoológica Mexicana (n. s.), 28(1): 133-144.

RESUMEN. Se realizó un inventario de enemigos naturales del chapulín (Orthoptera: Acridoidea) como resultado de observaciones hechas a través de la exploración, muestreos de ootecas, ninfas y adultos que se llevaron a cabo de 2002 a 2007 en 11,242 ha de 101 comunidades de 11 municipios del sur del Estado. Se examinaron 1620 ootecas y 8122 chapulines identificando tres especies de hongos entomopatógenos, un parasitoide y cinco depredadores. Se encontraron larvas de Epicauta spp. (Coleoptera: Meloidae) depredando ootecas de Melanoplus differentialis Thomas (Orthoptera: Acridoidea) y dos aves, Mimus polyglottos L. y Toxostoma curvirostre Swainson (Passeriformes: Mimidae) alimentándose de estadios ninfales o adultos de especies pequeñas. Se observaron estadios ninfales en la telaraña de Neoscona spp. (Araneae: Araneidae). Un depredador muy activo de ninfas pequeñas en las horas mas calientes del día fue el "moscón" Efferia spp. (Diptera: Asilidae). Se encontró a Sarcophaga spp. (Diptera: Sarcophagidae) en M. differentialis como huésped. Se observaron tres especies de hongos, Beauveria bassiana Balsamo (Hypocreales: Cordycipitaceae), Metharizium anisopliae Metschnikoff (Hypocreales: Clavicipitaceae) en todos los estadios y Entomophaga grylli Fresenius (Entomophthorales: Entomophthoraceae) en adultos de $M$. differentialis como hospedero, ocasionando epizootias con un buen nivel de infección.

Palabras clave: Muestreo, Inventario, Depredadores, Parasitoides, Patógenos.

Uribe-González, E. \& M. A. Santiago-Basilio. 2012. Contribution to the knowledge of natural enemies of the grasshopper (Orthoptera: Acridoidea) in the state of Querétaro, Mexico. Acta Zoológica Mexicana (n. s.), 28(1): 133-144.

ABSTRACT. We performed an inventory of natural enemies of grasshopper (Orthoptera: Acridoidea) as a result of observations made through exploration, sampling of egg pods, nymphs and adults were conducted from 2002 to 2007 in 11,242 ha of 101 communities in 11 municipalities of Southern Queretaro. 1620 egg pods were reviewed and 8122 grasshoppers; three species of entomophatogenis were identified, a parasitoid and five predators. Epicauta spp. (Coleoptera: Meloidae) larvae were found prey-

Recibido: 04/04/2011; aceptado: 26/10/2011. 
ing egg pods of Melanoplus differentialis Thomas (Orthoptera: Acridoidea) and two birds, Mimus polyglottos L. and Toxostoma curvirostre Swainson (Passeriformes: Mimidae) feeding nymphs or adults of small species. Nymphs were observed in the web of Neoscona spp. (Araneae: Araneidae). A very active predator of small nymphs during the hottest hours of the day was the blowfly Efferia spp. (Diptera: Asilidae). We found Sarcophaga spp. (Diptera: Sarcophagidae) in M. differentialis as a host. There were three species of fungus, Beauveria bassiana Balsamo (Hypocreales: Cordycipitaceae), Metharizium anisopliae Metschnikoff (Hypocreales: Clavicipitaceae) in all stages and Entomophaga grylli Fresenius (Entomophthorales: Entomophthoraceae) in adults of M. differentialis as a host, causing epizooties with a good level of infection.

Key words: Sampling, Inventory, Predators, Parasitoids, Pathogens.

\section{INTRODUCCIÓN}

El chapulín es la plaga económicamente más importante en el sur del estado de Querétaro, principalmente para cultivos de temporal (CESAVEQ 2011). Las especies más importantes por su cantidad son $M$. differentialis y Sphenarium purpurascens Charpentier (Orthoptera: Acridoidea) de 25 registradas para el estado (Salas-Araiza et al. 2006). El complejo de especies llamado chapulín tiene hábitos fitófagos muy variados, comen casi todo tipo de vegetal y en casos extremos de falta de material verde hacia el final de su ciclo biológico pueden comer o al menos roer desde papel hasta materiales de poliestireno expandido (unicel en México), comportamiento que se desconoce si su objetivo es cubrir algún requerimiento nutricional o es algún tipo de hábito (observación personal).

El cultivo del maíz es por excelencia el más importante en el estado; en el ciclo primavera-verano 2009 se cosecharon 76,385 ha de maíz grano (INEGI 2010), del que seguramente un $90 \%$ es de temporal. Con niveles de infestación por arriba del umbral económico y sin acciones de control, se estima de manera conservadora, que esta plaga puede ocasionar pérdidas en la producción de maíz y frijol en Querétaro superior a los 5 millones de pesos, sin considerar las pérdidas en la planta de maíz como forraje (CESAVEQ 2011).

En Querétaro, el chapulín se puede presentar desde junio después de las primeras lluvias con el temporal ya establecido, sin embargo, si este temporal se retrasa puede darse el caso que se presenten hasta el mes de agosto como sucedió en éste año (CESAVEQ 2011). En su estado natural se pueden observar diferentes estadios a la vez, pudiendo encontrar ninfas de primer estadio desde junio o julio hasta octubre, apareamientos de septiembre a diciembre, oviposturas de octubre a enero y muerte casi al $100 \%$ de la población en enero, sin embargo su población va disminuyendo sensiblemente por el frío principalmente desde octubre (CESAVEQ 2011).

Los controles químicos se llevan acabo año con año desde hace casi 10 como una medida de control preventiva de la población en sus sitios de eclosión en 5 mil a 8 mil ha por año, con el riesgo que conlleva el aplicar químicos en agostaderos, en particular por la toxicidad a animales que pastorean, fauna, insectos y peces. 
Por todo lo anterior, el objetivo principal de este trabajo fue identificar los géneros o especies de enemigos naturales del chapulín en sus diferentes estadios del ciclo biológico.

\section{MATERIALES Y MÉTODOS}

Área de estudio. Para el estudio solo se consideraron 11 de los 18 municipios del estado de Querétaro debido a que es donde se presenta el chapulín como plaga agropecuaria. Las comunidades donde se trabajo se encuentran entre $20^{\circ} 13^{\prime} 08.8^{\prime \prime}$ y $20^{\circ}$ 50 ' 41.3" de latitud Norte y $100^{\circ} 00^{\prime} 19.6^{\prime \prime}$ y $99^{\circ} 59^{\prime} 46.9^{\prime \prime}$ de longitud Oeste. Con altitudes que van de los $1800 \mathrm{msnm}$ en El Pueblito, municipio de Corregidora hasta los 2500 msnm en La Alameda del Rincón, municipio de Amealco; una precipitación promedio anual de $521 \mathrm{~mm}$ y una temperatura media anual de $19^{\circ} \mathrm{C}$. En la Figura 1 se indica el área de estudio.

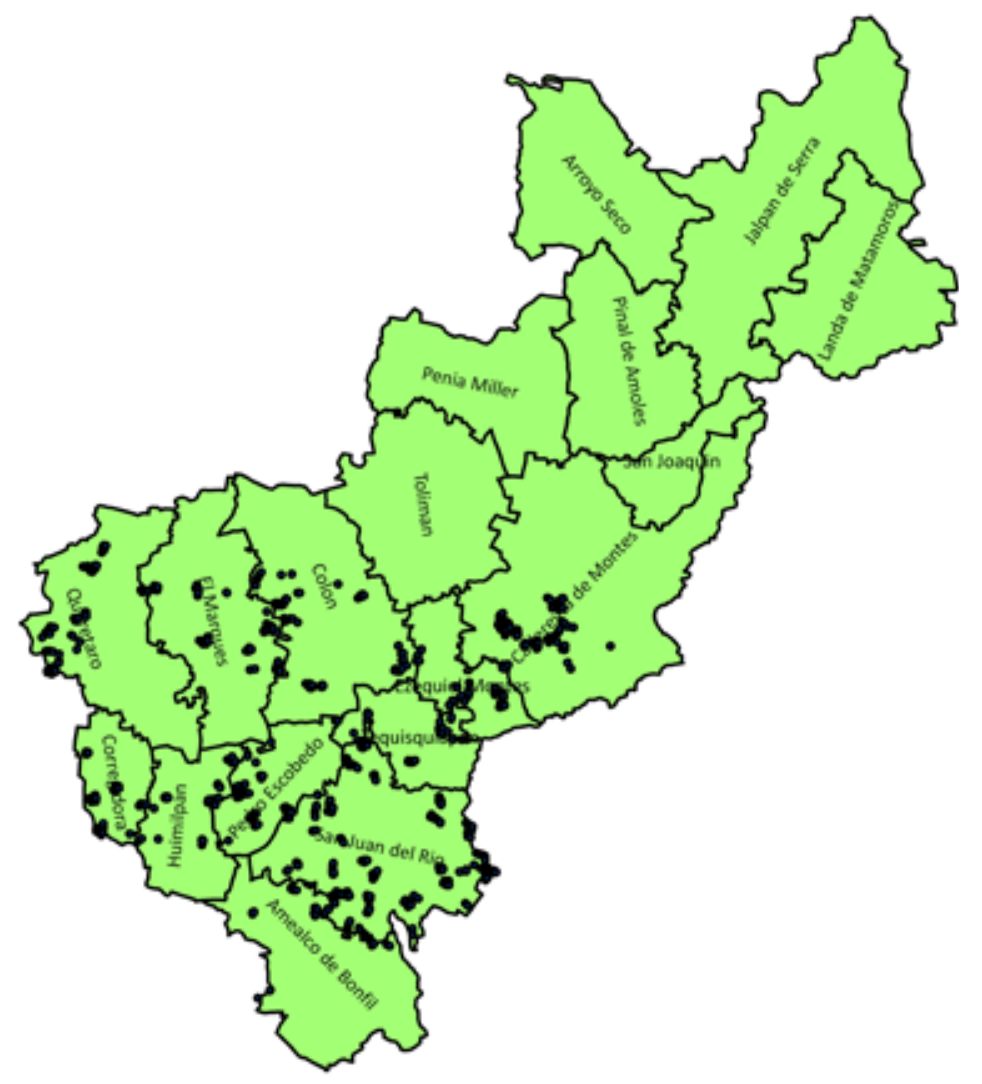

Figura 1. Sitios de muestreo y toma de muestras biológicas. 
Muestreo. Con el objeto de conocer los principales enemigos naturales del chapulín durante su ciclo biológico se registraron los hallazgos detectados de manera in situ durante el muestreo a través de la colecta directa u observación en su caso en microscopio estereoscópico (Carl Zeiss Modelo Stemi DV4), no se registró el nivel de ataque en el chapulín pues no formaba parte del objetivo de éste trabajo. La actividad se llevó a cabo en los perímetros de las parcelas, parcelas abandonadas, potreros, llanos y bordos de 2002 a 2007. El método de muestreo fue en "cinco de oros" tomando cinco muestras en superficies menores a 20 ha y 10 en mas de 20 ha.

Muestreo de ninfas y adultos. Las ninfas se muestrearon sobre la maleza de julio a septiembre usando una red de golpeo, los adultos se muestrearon de octubre a diciembre con red de golpeo u observación directa. Las muestras se depositaron en bolsas de papel de estraza, se congelaron y observaron en un microscopio estereoscópico. Durante el muestreo, de manera alterna, se estuvo vigilando el comportamiento de ejemplares de chapulín para detectar si alguno presentaba síntomas de parasitismo o enfermedad, así como observación de depredadores vertebrados.

Muestreo de ootecas. El muestreo se llevó acabo de enero a junio. Para ootecas se examinaron cepellones de $1 \mathrm{~m}^{2}$ por $5 \mathrm{~cm}$ en sitios donde se vio población de adultos en el muestreo. Se revisó con mucho cuidado cada terrón de tierra para detectar la mayor cantidad de ootecas. Estas se colectaron en frascos, se trasladaron al laboratorio y se revisaron minuciosamente en el microscopio estereoscópico para detectar síntomas de entomopatógeno, parasitismo o depredador.

Diagnóstico. En la identificación de patógenos y depredadores se usaron las claves descritas en Rees (1973); así mismo, se consideró el material fotográfico y descripción de síntomas detallado en Pfadt (2002). La depredación por meloidos fue confirmada por la presencia de larvas y pupas dentro de la ooteca o por las características de los agujeros en las paredes de las ootecas que significa daño por ataque (Popov et al. 1990; Latchininsky \& Launois-Luong 1992). Los depredadores vertebrados se identificaron in situ.

\section{RESULTADOS Y DISCUSIÓN}

En éste trabajo se compiló información de observaciones hechas en las diferentes actividades de vigilancia fitosanitaria de la campaña contra el chapulín de 2002 a 2007 en 101 comunidades de 11 municipios (Cuadro 1). En este cuadro se indica el área de estudio y la cantidad de muestras biológicas examinadas para la identificación de los enemigos naturales indicados en el Cuadro 2. En la Figura 1 se muestran los sitios georreferenciados del Cuadro 1 donde se han hecho actividades de vigilancia fitosanitaria y las identificaciones de los enemigos naturales que se enlistan en el Cuadro 2 
Cuadro 1. Material biológico examinado por superficie vigilada en el área de estudio de 2002 a 2007.

\begin{tabular}{lccccc}
\hline Municipio & Comunidades & $\begin{array}{c}\text { Superficie } \\
\text { Vigilada, ha }\end{array}$ & $\begin{array}{c}\text { Puntos } \\
\text { Georeferenciados }\end{array}$ & $\begin{array}{c}\text { Ootecas } \\
\text { examinadas }\end{array}$ & $\begin{array}{c}\text { Chapulines } \\
\text { examinados }\end{array}$ \\
\hline Amealco de Bonfil & 4 & 290 & 93 & 18 & 429 \\
Cadereyta de Montes & 7 & 594 & 123 & 102 & 199 \\
Colón & 14 & 1050 & 152 & 360 & 482 \\
Corregidora & 6 & 755 & 77 & 48 & 1447 \\
El Marques & 7 & 1350 & 82 & 96 & 410 \\
Ezequiel Montes & 3 & 200 & 47 & 60 & 572 \\
Huimilpan & 16 & 880 & 191 & 48 & 504 \\
Pedro Escobedo & 6 & 660 & 117 & 132 & 1063 \\
Querétaro & 10 & 435 & 117 & 72 & 1603 \\
San Juan del Río & 22 & 4588 & 272 & 540 & 1091 \\
Tequisquiapan & 6 & 440 & 71 & 144 & 322 \\
Suma & 101 & 11,242 & 1342 & 1620 & 8122 \\
\hline
\end{tabular}

y 3. Se identificaron tres especies de hongos entomopatógenos, un parasitoide y cinco depredadores, de estos últimos, dos son aves.

Los enemigos naturales del chapulín, como en el resto de insectos, están sujetos a un amplio número de parasitoides, depredadores y patógenos, incluyendo hongos, protozoarios y virus (Henry et al. 1985). En México, en la última década se han llevado acabo algunos trabajos tendientes a identificar los principales enemigos naturales del chapulín; destacan las publicaciones de Rivera (2004) en el Bolsón de Mapimí, Durango; Lozano-Gutiérrez \& España-Luna 2006(2009) en comunidades del municipio de Villanueva, Zacatecas; García-Gutiérrez y González-Maldonado 2006(2009) en el estado de Durango; Salas-Araiza \& Salazar-Solís 2006(2009) en el Bajío del estado de Guanajuato, principalmente en el municipio de Irapuato. Por lo observado en éste trabajo y lo descrito en los citados, se sabe que estos enemigos naturales ejercen cierta presión sobre el chapulín, sin embargo, no son suficientes para que mermen la población pues siempre ha sido necesario su control químico debido a que rebasan el umbral económico. Las epifitias observadas en relación a hongos, son casos especiales en las que no se detectaron mermas aparentes de la población, los depredadores y parasitoides también son casos aislados en los que se duda ocasionen un impacto considerable en la población de chapulín.

El hongo E. grylli, probablemente el patotipo 2 (Entomophaga calopteni) (Bidochka \& Roberts 2002), se observó afectando adultos de M. differentialis en el 39\% de las comunidades con registro de enemigos naturales (Cuadro 3). Se observaron ejemplares "momificados", en posición vertical, viendo hacia arriba y abrazados de 


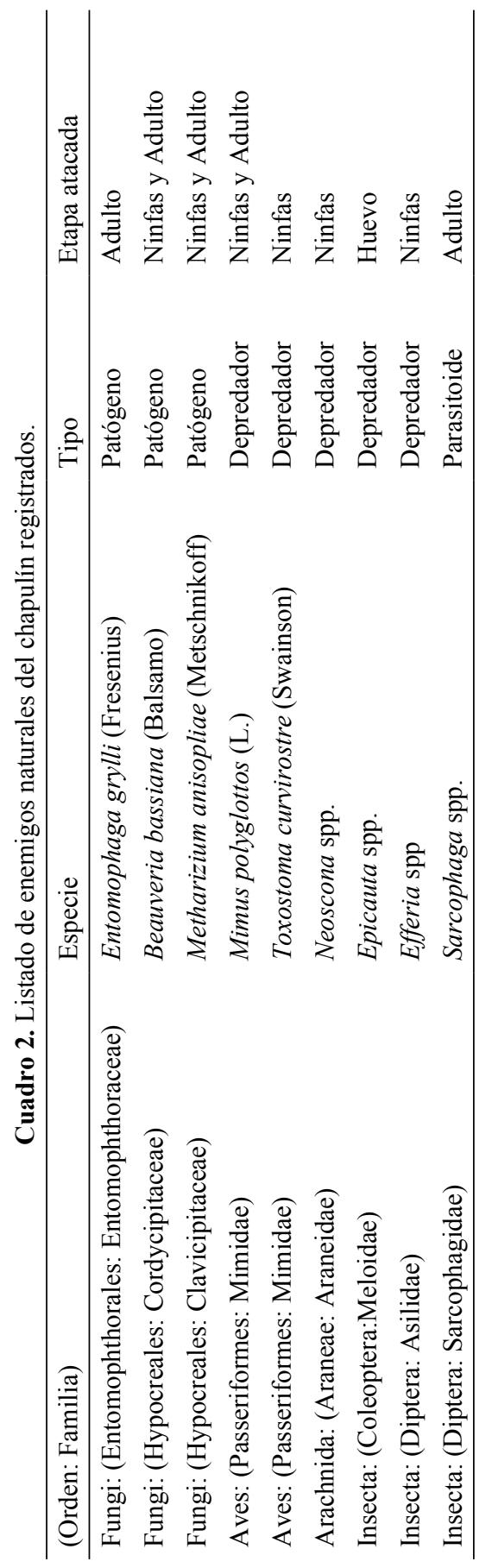


Cuadro 3. Comunidades con registro de enemigos naturales.

\begin{tabular}{|c|c|c|}
\hline Enemigo natural & $\begin{array}{l}\text { No. comunidades } \\
\text { (\% del total) }\end{array}$ & Municipio: comunidades \\
\hline $\begin{array}{l}\text { Entomophaga grylli } \\
\text { (Fresenius) }\end{array}$ & $20(39 \%)$ & $\begin{array}{l}\text { Pedro Escobedo: El Sauz, Ajuhitlancito, San Cirilo, } \\
\text { Escolásticas. San Juan del Río: Arcila y Paso de Mata } \\
\text { Huimilpan: Taponas, El Vegil, Lagunillas, Buenavista, } \\
\text { Ceja de Bravo y El Granjeno. Corregidora: Charco } \\
\text { Blanco, Bravo y Presa de Bravo. Querétaro: La Solana } \\
\text { y Montenegro. El Marqués: Atongo. Colón: El Saucillo. } \\
\text { Cadereyta de Montes: La Nopalera. }\end{array}$ \\
\hline $\begin{array}{l}\text { Beauveria bassiana } \\
\text { (Balsamo) }\end{array}$ & $1(2 \%)$ & Amealco de Bonfil: Los Arenales. \\
\hline $\begin{array}{l}\text { Metharizium } \\
\text { anisopliae } \\
\text { (Metschnikoff) }\end{array}$ & $5(10 \%)$ & $\begin{array}{l}\text { San Juan del Río: Arcila. Pedro Escobedo: } \\
\text { Ajuchitlancito. Tequisquiapan: Hacienda Grande. } \\
\text { Huimilpan: Buenavista y Taponas. }\end{array}$ \\
\hline $\begin{array}{l}\text { Mimus polyglottos } \\
\text { (L.) y Toxostoma } \\
\text { curvirostre } \\
\text { (Swainson) }\end{array}$ & $3(6 \%)$ & $\begin{array}{l}\text { San Juan del Río: Santa Rita. Pedro Escobedo: Dolores } \\
\text { de Ajuchitlancito. Colón: El Zamorano. }\end{array}$ \\
\hline Neoscona spp. & $? ? ? ?^{*}$ & Todos los municipios: más de 80 comunidades. \\
\hline Epicauta spp & $31(61 \%)$ & $\begin{array}{l}\text { Cadereyta de Montes: San Javier. Colón: El Zamorano, } \\
\text { Fracción El Moral y Galeras. Corregidora: Charco } \\
\text { Blanco, El Jaral y Joaquín Herrera. El Marqués: El } \\
\text { Lobo, Guadalupe la Venta, La Griega, Santa Cruz, Santa } \\
\text { María de los Baños y Tierra Blanca. Ezequiel Montes: } \\
\text { El Bondotal, Loberas y Palo Seco. Huimilpan: Los Cues } \\
\text { y Taponas. Pedro Escobedo: Ajuchitlancito, Dolores de } \\
\text { Ajuchitlancito y La Venta. Querétaro: Buenavista, El } \\
\text { Gachupín y Mompaní. San Juan del Río: Estancia de } \\
\text { Santa Lucía, La Llave, Ojo de Agua, San Sebastián de } \\
\text { las Barrancas, Santa Bárbara la Cueva, Santa Isabel El } \\
\text { Coto. Tequisquiapan: San José la Laja. }\end{array}$ \\
\hline Efferia spp. & $1(2 \%)$ & Pedro Escobedo: Escolásticas. \\
\hline Sarcophaga spp. & $2(4 \%)$ & Pedro Escobedo: Escolásticas. Huimilpan: La Peña. \\
\hline
\end{tabular}

*No se tiene un registro exacto.

las partes más altas de pastos, herbáceas o arbustivas. Se reporta que dos patotipos son nativos de Norte América y uno más que se aisló de una especie de Australia (Bidochka \& Roberts 2002). En México se han publicado algunos trabajos sobre éste patógeno (Sánchez-Peña et al. 1996; Sánchez-Peña 2000, 2005; Sánchez-Peña \& Álvarez-Amador 2001, 2003), destacando por su importancia el de Sánchez-Peña (2005) debido a que trabajó sobre la producción in vitro de hifas como uso potencial 
para la obtención de conidias.

Con relación a los hongos del orden Hypocreales solo encontramos adultos de Sphenarium purpurascens infectados con Beauveria bassiana en la comunidad de Los Arenales del Municipio de Amealco de Bonfil y M. differentialis infectados por M. anisopliae en el 10\% de las comunidades con registro de enemigo natural (Cuadro $3)$. En los ejemplares enfermos se les ha observado un crecimiento miceliar en partes de su cuerpo o cubriéndolo por completo; micelio de color blanco en B. bassiana o de color verde con M. anisopliae (Bidochka \& Roberts 2002). Estos hongos son los agentes de control biológico más investigados en el mundo, pero recientemente se reporto a Fusarium verticillioides (Saccardo) Nirenberg (Ascomycota: Hypocreales) infectando de manera natural ninfas y adultos de Tropidacris callaris (Stoll) (Orthoptera: Acridoidea: Romaleidae) en la Provincia del Chaco en Argentina (Pelizza et al. 2011). En México se han hecho algunos trabajos con ciertas variedades de B. bassiana y M. anisopliae (Rodríguez del Bosque \& Arredondo-Bernal 2007). Destacan los trabajos del equipo de Ludivina Barrientos Lozano del Instituto Tecnológico de Cd. Victoria, Tamaulipas, México con M. anisopliae var. acridum contra langosta Schistocerca piceifrons piceifrons Walker (Orthoptera: Acrididae), el equipo de Cipriano García Gutiérrez del CIIDIR-IPN Unidad Sinaloa, México con B. bassiana sobre Brachystola magna (Girard) (Orthoptera: Romailedae), el equipo de Raquel Alatorre Rosas del Instituto de Fitosanidad del Colegio de Postgraduados en Montecillos, Texcoco, México con B. bassiana contra S. purpurascens (Orthoptera: Pyrgomorphidae), el equipo de Víctor Manuel Hernández Velázquez del Centro de Investigación en Biotecnología de la Universidad Autónoma del estado de Morelos con M. anisopliae var. acridum contra langosta $S$. piceifrons piceifrons.

En México, se sabe que el Centro Nacional de Referencia de Control Biológico contaba hasta 2002 con una colección de 40 cepas de Metarhizium spp., destacando tres cepas por su virulencia (Barrientos-Lozano et al. 2002). Estos mismo autores mencionan que por análisis de ADN dos de las tres cepas mexicanas (MaPL40 y MaPL32) son similares a una cepa Australia (FI-985), sugiriendo que son la misma variedad. Metarhizium anisopliae var. acridum se encuentra de manera comercial en Australiana como Green Guard ${ }^{\circledR}$ (Hunter 2005) y Metarhizium acridum en África como Green Muscle ${ }^{\circledR}$ (Mullié \& Youssoupha 2010), en el primer país se ha utilizado desde el año 2000 con buenos resultados en el control de cuatro especies de Acridoideos (Milner \& Hunter 2001) en alrededor de 100,000 ha que representa hasta un $10 \%$ de la superficie tratada con Manejo Integrado de la Plaga (MIP) (Hunter 2010), no obstante, la temperatura puede ser una limitante en la efectividad de $B$. bassiana (Amarasekare \& Edelson 2004 y Hunter 2010).

El chapulín es también parte de la dieta de Mimus polyglottos L. y Toxostoma curvirostre Swainson (Passeriformes: Mimidae); estas aves se han visto depredando solo en un $6 \%$ de las comunidades con registro de enemigo natural (Cuadro 3). A 
estas aves se les puede ver en puestos de vigilancia, preferentemente en lo alto de matorrales, observan al insecto, vuelan, lo capturan y lo comen en el mismo lugar o vuelan a su punto de vigilancia para devorarlo (observación personal). Ambas especies consumen ninfas, pero a $M$. polyglottos se le ha visto consumiendo adultos de algunas especies pequeñas que al volar le llaman la atención y las captura (observación personal). Las aves son importantes depredadores del chapulín a las que poca atención se les ha dado; Branson (2005) y Mullié \& Youssoupha (2010) coinciden al citar una marcada preferencia por chapulines de talla grande y media en sus aves estudiadas (talla grande), mismas que no se les ha visto en nuestra área de estudio. No obstante, McEwen et al. (2002) hacen referencia a tres aves que pueden consumir hasta 100 chapulines por día, entre ellas a Sturnella spp. (Passeriformes: Icteridae) que pertenece al mismo orden que las mencionadas en este estudio. En relación a otros vertebrados, se sabe que el chapulín es un insecto importante en la dieta del coyote Canis latrans Say (Carnívora: Canidae) (Guerrero et al. 2002) y éste es muy abundante en ésta zona pero no se consideró el estudio de sus heces para determinar consumo en nuestra área de estudio, sin embargo, se ha observado el consumo ocasional de adultos de B. magna por perros Canis lupus familiaris L. (Carnívora: Canidae) (observación personal).

La araña Neoscona spp. (Araneae: Araneidae) necesita de las arbustivas de los matorrales o herbáceas leñosas para hilar su telaraña, por esta razón, son en éstas áreas donde se les encontró depredando a las ninfas principalmente cuando el maíz y la maleza están en floración. El chapulín salta accidentalmente a la telaraña donde es capturado por el depredador, quien inmediatamente lo "enreda" con telaraña para después comerlo. Se les ha visto en las zonas cerriles de más de 80 comunidades de los municipios donde se trabajó (Cuadro 3). Las arañas son de los depredadores menos estudiados, se sabe de la existencia de cuando menos nueve especies, pero la lista es probable esté incompleta (Salas-Araiza \& Salazar-Solís, 2006(2009)). Estos mismos autores reportan la presencia del género Argiope (Araneae: Araneidae) en el municipio de Irapuato, Guanajuato, México depredando ninfas del género Syrbula (Orthoptera: Acridoidea) y Rivera (2004) reporta en el Bolsón de Mapimí, Durango, México la presencia de Araneus diadematus? (Araneae: Araneidae), Argiope aurentia Lucas (Araneae: Argiopidae) y Aphonopelma spp. (Araneae: Theraphosidae).

La borreguilla Epicauta spp. (Coleoptera: Meloidae) en su fase de larva se le ha visto depredando ootecas en varias comunidades (Cuadro 3). En ootecas detectadas durante los muestreos en 90 comunidades se observaron signos de depredación por éste coleóptero en 31 de ellas ( $21.38 \%$ de depredación), detectando también que prefiere en un $98 \%$ las ootecas de Melanoplus spp., quizás por su abundancia o tamaño y solo en un 2\% las de B. magna (Datos no publicados). La borreguilla es un coleóptero que en su fase larval es un importante depredador de ootecas de chapulín en Estados 
Unidos de América, la hembra adulta deposita masas de huevos cercas de las ootecas, cuando nacen las larvas buscan las ootecas, las perforan y devoran los huevecillos, si aun no completa su desarrollo larval sigue buscando más ootecas (Capinera 2003); su ciclo biológico puede durar hasta dos años, según describe Rees (1973). La presencia de Epicauta spp. ha sido reportada por otros investigadores en México; Rivera (2004) indica que es un depredador de ootecas de Bootettix argentatus Bruner (Orthoptera: Acrididae) y Lozano-Gutiérrez \& España-Luna (2006(2009)) en ootecas de Brachystola spp.

La mosca Sarcophaga spp. se ha visto parasitando en la Comunidad de Escolásticas, Pedro Escobedo y en La Peña, Huimilpan. Si bien es cierto que la mayoría de larvas de especies de sarcofágidos se alimentan de materia en descomposición, se han detectado algunas especies parásitas; Lozano-Gutiérrez \& España-Luna (2006(2009)) encontraron que la preferencia de parasitar entre macho y hembra de Brachystola spp. es similar, no obstante, se encontraron hasta nueve larvas por hembra contra tres por macho; esto quizás se deba al tamaño de la hembra. En Estados Unidos se reportan de 21 a 23 especies parásitas de chapulín (Hostetter 2002), cinco de las cuales son las más importantes por su nivel de parasitismo hasta del 50\%. Las moscas de la familia Sarcophagidae son ovovivíparos, sin excepción, lo que significa que sus huevos eclosionan en el útero y la hembra depositan una larva viva en el hospedero (Shewell 1987).

Por su parte, el moscón Efferia spp. (Diptera: Asilidae) se ha visto depredando ninfas en Escolásticas municipio de Pedro Escobedo. Rees \& Onsager (1985), mencionan que este insecto es muy voraz y captura al chapulín en pleno vuelo, además, de las 856 especies reportadas 26 depredan chapulín y solo seis tienen definida su preferencia por este insecto. Mientras tanto, Lavigne \& Pfadt (1966), concluyeron en un trabajo de seis años en Wyoming, Estados Unidos de América que son tres las especies que se alimentan exclusivamente de chapulín y pueden reducir la población de un 11 a un $15 \%$.

Los enemigos naturales del chapulín en las áreas estudiadas son diversos y contribuyen en su control y regulación de poblaciones, sin embargo sus bandadas llegan a ser tan grandes en la mayoría de comunidades que hace necesario su control químico a través de aplicaciones localizadas en sitios de eclosión.

Agradecimientos. Al Dr. Manuel Darío Salas Araiza del Instituto de Ciencias Agrícolas de la Universidad Autónoma de Guanajuato por su ayuda en la identificación de enemigos naturales.

\section{LITERATURA CITADA}

Amarasekare, K. G. \& J. V. Edelson. 2004. Effect of temperature on efficacy of insecticides to differential grasshopper (Orthoptera: Acrididae). Journal of Economic Entomology, 97: 1595-1602. 
Barrientos-Lozano, L., V. M. Hernández-Velázquez, R. J. Milner \& D. M. Hunter. 2002. Advances in biological control of locusts and grasshoppers in México. Journal of Orthoptera Research, 11: 77-82.

Bidochka, M. J. \& D. W. Roberts. 2002. I. Biological Control: I.5 Identification of Fungal Pathogens of Grasshoppers, pp. I.5-1-I.5-2. In: R. E. Pfadt (Ed.). Field Guide to Common Western Grasshoppers. Wyoming Agricultural Experiment Station. Bulletin 912, Third Edition.

Branson, D. H. 2005. Direct and indirect effects of avian predation on grasshopper communities in northern mixed-grass prairie. Environmental Entomology, 34: 1114-1121.

Capinera, J. L. 2003. Striped blister beetle. Epicauta vittata (Fabricius) (Coleoptera: Meloidae). University of Florida. IFAS Extension.

[CESAVEQ] Comité Estatal de Sanidad Vegetal de Querétaro, A.C. 2011. Información Técnica de la Campaña Contra el Chapulín. Calamanda de Juárez, El Marques, Querétaro, México, s/p.

García-Gutiérrez, C. \& M. B. González-Maldonado. 2006 (2009). Control biológico de la plaga del chapulín (Orthoptera: Acrididae) en Durango, México. Vedalia, 13: 79-83.

Guerrero, S., M. H. Badii, S. S. Zalapa \& A. E. Flores. 2002. Dieta y nicho de alimentación del coyote, zorra gris, mapache y jaguarundi en un bosque tropical caducifolio de la costa sur del estado de Jalisco, México. Acta Zoológica Mexicana (n. s.), (86): 119-137.

Henry, J. E., M. C. Wilson, E. A. Oma \& J. L. Fowler. 1985. Pathogenic micro-organisms isolated from West African grasshoppers (Orthoptera: Acrididae). Tropical Pesticide Management, 31: 192195.

Hostetter, D. L. 2002. I. Biological Control: I.8 Natural Enemies Attacking Grasshopper Nymphs and Adults, pp. I.8-1-I.8-7. In: R. E. Pfadt (Ed.). Field Guide to Common Western Grasshoppers. Wyoming Agricultural Experiment Station. Bulletin 912, Third Edition.

Hunter, D. M. 2005. Mycopesticides as part of integrated pest management of locusts and grasshoppers. Journal of Orthoptera Research, 14: 197-201.

Hunter, D. M. 2010. Credibility of an IPM approach for locust and grasshopper control: The Australian example. Journal of Orthoptera Research, 19: 133-137.

[INEGI] Instituto Nacional de Estadística, Geografía e Informática. 2010. Anuario Estadístico del Sector Rural. Sistema Nacional de Información para el Desarrollo Rural Sustentable. Secretaría de Desarrollo Agropecuario en Querétaro.

Latchininsky, A. V. \& M. H. Launois-Luong. 1992. Le Criquet marocain, Dociostaurus maroccanus (Thunberg, 1815), dans la partie orientale de son aire de distribution. CIRAD-GERDAT-PRIFAS, Montpellier, France, 270 pp.

Lavigne, R. J. \& R. E. Pfadt. 1966. Parasites and predators of Wyoming rangeland grasshoppers. Monogr. 3. Laramie, WY: University of Wyoming and Wyoming Agricultural Experiment Station.

Lozano-Gutiérrez, J. \& M. P. España-Luna. 2006 (2009). Enemigos naturales y control biológico de Brachystola magna Girard y Brachystola mexicana Bruner (Orthoptera: Acrididae) con Beauveria bassiana en Zacatecas, México. Vedalia, 13: 91-96.

McEwen, L. C., B. E. Petersen \& C. M. Althouse. 2002. I. Biological Control: I.10 Birds and Wildlife as Grasshopper Predators, pp. I.10-1-I.10-4. In: R. E. Pfadt (Ed.). Field Guide to Common Western Grasshoppers. Wyoming Agricultural Experiment Station. Bulletin 912, Third Edition.

Milner, R. J. \& D. M. Hunter. 2001. Recent developments in the use of fungi as biopesticides against locusts and grasshoppers in Australia. Journal of Orthoptera Research, 10: 271-276.

Mullié, W. C. \& G. Youssoupha. 2010. Does bird predation enhance the impact of Green Muscle ${ }^{\circledR}$ (Metarhizium acridum) used for grasshopper control? Journal of Orthoptera Research, 19: 139155. 
Pelizza, S. A., S. A. Stenglein, M. N. Cabello, M. I. Dinolfo \& C. E. Lange. 2011. First Record of Fusarium verticillioides as an Entomopathogenic Fungus of Grasshoppers. Journal of Insect Science, 70: 1-8.

Pfdat, R. E. 2002. Field guide to common western grasshoppers. Wyoming Agricultural Experimental Station. Bulletin, 912, Third Edition.

Popov, G. B., M. H. Launois-Luong \& J. J. van der Weel. 1990. Les oothèques des criquets du Sahel. Niamey, Collection Acridologie Opérationnelle No. 7. Ministère dos Affaires Etrangères des PaysBas et CIRAD/PRIFAS.

Rees, N. E. \& J. A. Onsager. 1985. Parasitism and survival among rangeland grasshoppers in response to suppression of robber fly (Diptera: Asilidae) predators. Environmental Entomology, 14: 20-23.

Rees, N. E. 1973. Arthropod and nematode parasites, parasitoids, and predators of Acrididae in America north of Mexico. Tech. Bull. 1460. Washington, DC: U.S. Department of Agriculture, Agricultural Research Service.

Rivera G., E. 2004. Records of predators and parasites (vertebrates and invertebrates) of creosote bush grasshopper Bootettix argentatus Bruner, 1889 (Orthoptera: Acrididae: Gomphocerinae) from the Bolsón de Mapimí, Dgo. (Chihuahuan Desert), México. Acta Zoológica Mexicana (n. s.), 20: 287290.

Rodríguez del Bosque, L. A. \& H. C. Arredondo-Bernal. 2007. Bibliografía Sobre Control Biológico en México hasta 2005. 02 Jul. 2010. <http://www.controlbiologico.org.mx/Bibliografia.htm>.

Salas-Araiza, M. D. \& E. Salazar-Solís. 2006 (2009). Enemigos naturales de la plaga del chapulín (Orthoptera: Acrididae) con énfasis en Guanajuato, México: Una breve revisión. Vedalia, 13: 57-64.

Salas-Araiza, M. D., P. Alatorre-García \& E. Uribe-González. 2006. Contribución al conocimiento de los Acridoideos (Insecta: Orthoptera) del estado de Querétaro, México. Acta Zoológica Mexicana (n. s.), 22: 33-43.

Sánchez-Peña, S. \& S. Álvarez-Amador. 2001. Potencial del complejo Entomophaga grylli (Fres.) Batko (Zygomycetes: Entomophthorales) para el control biológico de algunos acrídidos (Orthoptera), pp. 49-51. In: DGSV (Ed.). Memoria del Taller de Control Microbiano de Langosta. Dirección General de Sanidad Vegetal. 2-5 de octubre de 2001, Mérida, Yucatán, México.

Sánchez-Peña, S. 2000. Entomopathogens from two Chihuahuan desert localities in Mexico. BioControl, 45: 63-78.

Sánchez-Peña, S. R. \& S. Álvarez-Amador. 2003. Potencial del complejo Entomophaga grylli (Fres.) Batko (Zygomycetes: Entomophthorales) para el control biológico de algunos acrídidos (Orthoptera) en México. Vedalia, 9-10: 69-72.

Sánchez-Peña, S., R. Carruthers, M. Lynn-Cummins \& J. C. Stephen-Wraight. 1996. Entomopatógenos como agentes de control biológico clásico, experiencias con Entomophaga grylli (Entomophthorales) contra saltamontes y langostas. Memoria del XIX Congreso Nacional de Control Biológico. Sociedad Mexicana de Control Biológico. 14-15 de Noviembre de 1996, Culiacán, Sinaloa, México. pp. 8-10.

Sánchez-Peña, S. R. 2005. In Vitro production of hyphae of the grasshopper pathogen Entomophaga grylli (Zygomycota: Entomophthorales): Potential for production of conidia. Florida Entomologist, 88: 332-334.

Shewell, G. E. 1987. Sarcophagidae, pp. 1159-1186. In: J. F. McAlpine et al. (Eds). Manual of Nearctic Diptera. vol. 2. Monogr. 28. Lethbridge, CN: Agriculture Canada, Research Branch, Biosystematics Research Center. 